\title{
Modeling Diameter Growth and Self-Thinning in Planted Sugi (Cryptomeria japonica) Stands
}

\author{
Tohru Nakajima $^{*}, 1$, Mitsuo Matsumoto ${ }^{2}$ and Norihiko Shiraishi ${ }^{3}$ \\ ${ }^{1}$ Laboratory of Global Forest Environmental Studies, Graduate School of Agricultural and Life Sciences, the University \\ of Tokyo, 1-1-1 Yayoi, Bunkyo-ku, Tokyo 113-8657, Japan \\ ${ }^{2}$ Forestry and Forest Products Research Institute, 1 Matsunosato, Tsukuba 305-8687, Japan \\ ${ }^{3}$ Laboratory of Forest Management, Graduate School of Agricultural and Life Sciences, University of Tokyo 1-1-1 \\ Yayoi, Bunkyo-ku, Tokyo 113-8657, Japan
}

\begin{abstract}
The objectives of this study were to analyze diameter growth in relation to natural thinning in high-density stands in even-aged, pure plantation forests and to develop a growth prediction system based on Japanese permanent plot data. Long-term data recorded in even-aged, pure, unthinned stand plots of Sugi (Cryptomeria japonica) forests were available. Relationships between log-transformed average diameter at breast height (DBH) and stand density were analyzed. In addition, the self-thinning ratio $(S T R)$ was analyzed from data gathered from unthinned permanent plots. Relationships between $S T R$ and the yield index, $R y$ (the ratio of actual stand volume to that at full stand density), were also analyzed and modeled. Based on these analyses, the diameter growth rate was formalized as a function of DBH, stand density and stand age, using parameters derived from full density curves. Goodness of fit of predictions of diameter growth in unthinned stands using the estimated parameters were evaluated by comparing predicted stand density and DBH with those observed in the permanent plots. The average error rate, derived by averaging the absolute values of all calculated error rates for the estimated stand density, was $1.8 \%$ with a maximum of $6.4 \%$. The average error rate for the DBH was $2.9 \%$, with a maximum of $8.1 \%$. The squared Pearson's correlation coefficients of predicted and observed average DBH were between 0.97 and 1.0.
\end{abstract}

Keywords: Cryptomeria japonica plantation, full density curve, growth model, self-thinning.

\section{INTRODUCTION}

Planning, decision making and the implementation of sound silvicultural practices in forest management require accurate predictions of how stand condition and density are related and likely to change over time [1]. High quality timber production has mainly relied on silvicultural practices in managed stands, such as controlling stand density by thinning. It is also important to be able to predict growth rates in unthinned stands, and the quantity of dead-wood in them in order to make estimates of carbon stocks. Unthinned stands have long been studied in many parts of the world, including the United States [2], Europe [3, 4], and Asia [1, 5].

Generally, two kinds of models are available for analyzing and predicting growth in unthinned stands: process [6] and empirical $[7,8]$ models. The aim of process models is to use knowledge of underlying ecological processes to simulate future forest resources. In contrast, empirical models can be used in conjunction with traditional forest mensuration to analyze the relationships between variables such as stand growth, self-thinning and stand density [8-11].

*Address correspondence to this author at the Laboratory of Global Forest Environmental Studies, Graduate School of Agricultural and Life Sciences, the University of Tokyo, 1-1-1 Yayoi, Bunkyo-ku, Tokyo 113-8657, Japan; Tel: +81-3-5841-5708; Fax: +81-3-5841-5235;

E-mail: nakajima@fr.a.u-tokyo.ac.jp
Since forest owners and managers need to collect less data in order to implement recommendations based on empirical models, past studies have often been based on empirical concepts such as the allometric relationship underlying full density curves, i.e. curves describing the correlation between the number of trees per unit area in a full density (unthinned) stand and their average diameter [12-14].

Early studies on self-thinning in high-density Japanese stands in which empirical models were employed [15] often used stand density control diagrams [16-20] based on full density curves [21]. However, these studies were all conducted between 14 and 49 years ago, when Japanese timber prices were higher and standard Japanese even-aged pure stands could be managed by relatively frequent thinning using standard density-control curves [22, 23], although it was known that some data sets used to derive full density curves were inadequate [21]. Since the cited studies were conducted, further data on high-density stands have accumulated through the periodic monitoring of unthinned permanent plots [24], i.e. experimental plots in the area of interest (generally known as silvicultural trials in the USA), used to collect time series of data [25]. Nevertheless, these earlier studies on permanent plots can provide benchmarks that can be used to evaluate more recently calculated full density curves. Although more than half of the planted forests in Japan are over 40 years old, their rotation period has been extended by reduced logging [26]. It is therefore important to investigate thoroughly our ability to predict 
stand growth over the long-term, under high density conditions.

Some process models that have been developed using information obtained from unthinned stands in Japan enable us to predict stand biomass, litter fall, and net primary production at the level of individual trees, by using meteororogical and biological data [5]. However, it would also be useful if forest managers were able to predict diameter growth and stand density from full density curves applicable at the stand level, because Japanese timber prices are critically dependent on $\log$ diameter [27]. Moreover, declining timber prices discourage forest owners from managing planted forests, causing a decline in thinning recent studies $[28,29]$ have suggested that more than half of all Japanese even-aged, pure planted stands have not been thinned for at least 10 years - accompanied by increases in the abundance of unmanaged, even-aged, pure forests [26]. Before timber prices fell, in line with the prevailing economic conditions in Japan, many areas of planted forests were frequently thinned according to standard density controls based on standard density curves [23]. However, since there are now increasing areas of unthinned stands in planted forests in Japan [28], it seems reasonable to predict diameter growth in these high density stands using full density curves.

It is therefore important for estimating both timber production and the carbon sink value of Japanese planted forests, to be able to predict growth in high-density stands where no thinning is being undertaken.

The objective of the present study was to test the hypothesis that average diameter growth could be estimated by formalizing a function of existing $\mathrm{DBH}$, stand density and stand age, using parameters derived from full density curves, and to develop a growth prediction system based on permanent plot data obtained from planted forests in Japan.

\section{MATERIALS AND METHODS}

\section{Study Area}

The University of Tokyo Chichibu Forest $\left(35^{\circ} 55^{\prime} \mathrm{N}\right.$, $138^{\circ} 53^{\prime}$ E) is in Chichibu municipality, Saitama Prefecture, Japan, between $530 \mathrm{~m}$ and $1,980 \mathrm{~m}$ above sea level. The terrain is undulating with steep slopes, and most soils are of the brown forest type. The forest is located in a cooltemperate zone, with an average annual temperature of 10.7 ${ }^{\circ} \mathrm{C}$, and average annual rainfall of $1,294 \mathrm{~mm}$. The total forest area is 5,812 ha, of which 3,101 ha $(53 \%)$ are secondary forests covered by mainly deciduous broadleaved trees, and 1,889 ha $(33 \%)$ are primary forest. The remaining 767 ha $(13 \%)$ are planted forest, of which 169 ha $(22 \%)$ are Sugi (Cryptomeria japonica) stands, 291 ha (38\%) are Hinoki (Chamaecyparis obtusa) stands, 207 ha $(27 \%)$ are Karamatsu (Larix leptolepis) stands, the remaining 100 ha $(13 \%)$ are Sawara (Chamaecyparis pisifera) and other planted forest. Since 1955, many permanent research plots have been established in plantations of Sugi, which is the most common tree species in even-aged pure stands in Japan. Although most of the planted stands have not had repeated ground surveys conducted in them, the plots listed in Table 1 have especially long series of measurement records.

Tree heights, DBH, and stand density (stems per ha) in unthinned stands have been recorded approximately every 5 years in eight permanent plots: Yatakesawa $\mathrm{C} 1$ (0.2387 ha), Yatakesawa C2 $(0.0710 \mathrm{ha})$, Iriyama C3 (0.1280 ha), Yohkurasawa C4 (0.0502 ha), Idosawa V1 (0.0957 ha), Kosuberisawa V2 (0.1234 ha), Yohkurasawa V3 (0.0369 ha), and Iriyama V4 (0.0554 ha) [24] (Table 1). The permanent plots are rectangular. The potential vegetation and vegetation cover of these plots is herbal type. These data were partitioned for model calibration (C1-C4 of plot ID) and validation (V1-V4 of plot ID).

\section{Methodology}

We investigated the relationships between stand growth, self-thinning and stand density. The arithmetic mean of DBH $(\mathrm{cm})$ and the stand density (stems/ha) were calculated for each set of records from the permanent plots, and analyzed with reference to past studies [21]. Reineke suggested that the number of trees per ha at full density varies with the average trunk diameter in the stand [13]. Based on this hypothesis, the curve describing the relationship between maximum number of trees per ha and average diameter, plotted on a common log-log scale, is defined as the full density curve in this study (formula (1)).

The parameters $d$ and $K$ in formula (1) were estimated by linear least-squares regression of log-transformed DBH against stand density. Since the study sites had not been

Table 1. Current Characteristics of the Permanent Plots as Determined by Ground Survey

\begin{tabular}{|c|c|c|c|c|c|c|c|c|c|}
\hline $\begin{array}{l}\text { Plot } \\
\text { ID }\end{array}$ & $\begin{array}{l}\text { Altitude } \\
\text { (m) }\end{array}$ & $\begin{array}{l}\text { Stand Age } \\
\text { (yr) }\end{array}$ & $\begin{array}{l}\text { Stand Density } \\
\text { (Stems/ha) }\end{array}$ & $\begin{array}{l}\text { Stand Height } \\
\text { (m) }\end{array}$ & Slope & Aspect & $\begin{array}{c}\text { Area } \\
\left(\mathbf{m}^{2}\right)\end{array}$ & $\begin{array}{c}\text { Number of } \\
\text { Measurements }\end{array}$ & $\begin{array}{c}\text { Total Basal Area } \\
\left(\mathrm{m}^{2} \mathrm{ha}^{-1}\right)\end{array}$ \\
\hline $\mathrm{C} 1$ & 950 & 69 & 1123 & 28.8 & Steep & SE & 2387 & 9 & 110.0 \\
\hline $\mathrm{C} 2$ & 950 & 69 & 1183 & 31.3 & Steep & SE & 710 & 11 & 112.0 \\
\hline $\mathrm{C} 3$ & 800 & 74 & 953 & 29.1 & Steep & SE & 1280 & 8 & 91.0 \\
\hline $\mathrm{V} 2$ & 940 & 72 & 1173 & 30.9 & Medium & E & 1234 & 7 & 104.9 \\
\hline V3 & 760 & 64 & 1197 & 28.5 & Medium & SW & 369 & 9 & 83.3 \\
\hline V4 & 850 & 73 & 1227 & 29.8 & Steep & S & 554 & 6 & 118.7 \\
\hline
\end{tabular}


thinned or disturbed by any natural catastrophe since the establishment of the permanent plots, it was assumed that formula (1) represented the full density curve for these sites.

$\log N=K+d \log D$

where: $D=\mathrm{DBH}(\mathrm{cm}) ; N=$ stand density (stems/ha); and $d$ $\& K$ are parameters.

The parameter values estimated in the present study were compared with full density curves derived from previous studies [21].

The self-thinning ratio $(S T R)$ of stems was also investigated. Following previous studies $[16,17,30]$, we analyzed the relationship between $S T R$ and the yield index $(R y)$, the latter being the ratio of the actual stand volume to that at full stand density. $R y$ is a relative measure of stand density: a ratio between current stand volume and maximum stand volume, ranging from 0 to 1 . The higher the value of $R y$, the higher the density of the stand and the greater the competition between trees.

$R y$ was plotted against the annual STR for increments of $R y\left(\mathrm{yr}^{-1}\right) . R y$ was estimated by substituting the parameters derived from a stand density management diagram [31], average stand height, and stand density, into the following formula (2) [30].

$$
R y=\frac{V}{V_{R f}}=\frac{a H^{b}+\frac{a^{\prime} H^{b^{\prime}}}{a^{\prime \prime} H^{b^{\prime \prime}}}}{a H^{b}+\frac{a^{\prime} H^{b^{\prime}}}{N}}
$$

where: $R y=$ yield index; $V=$ stand volume $\left(\mathrm{m}^{3} / \mathrm{ha}\right) ; V_{R f}=$ full stand volume $\left(\mathrm{m}^{3} / \mathrm{ha}\right) ; H=$ average stand height $(\mathrm{m}) ; N=$ stand density (stems/ha); and $a, a^{\prime}, a^{\prime \prime}, b, b^{\prime}, b^{\prime \prime}$ are parameters.

Following previous studies [30, 31], values of 0.072 , $1.374,5062,-2.87,234935,-1.496$ were substituted for the parameters $a, a^{\prime}, a^{\prime \prime}, b, b^{\prime}$ and $b^{\prime \prime}$ in formula (2). The decrease in stand density was estimated from a curve derived from the exponential function in formula (3), relating $R y$ to the selfthinning ratio [32]. The parameters in formula (3) were estimated by applying the exponential curve to the relationships between STR and Ry using the nonlinear leastsquares method that is called the quasi-Newton method, which is applicable to nonlinear relationships [38].

$$
S T R=A e^{B R y}
$$

where: $S T R=$ annual self-thinning ratio per increasing of $R y$ $\left(\mathrm{yr}^{-1}\right)$; and $A \& B$ are parameters.

The following assumptions were made in this study. First, previous studies [33,34] confirmed that stand growth could be estimated as a function of stand age. On this basis, it was assumed that, based on the full stand density curve, DBH would increase according to a growth function with fixed parameters. Previous studies $[33,34]$ made use of the Richards or Gompertz functions for stand growth prediction. Here, the Gompertz function, as given in formula (4), was selected for estimating parameter values to predict $\mathrm{DBH}$ growth because of its robustness and its simplicity.

$r=m \exp (-n t)$ where: $r=$ growth rate for average DBH $(\% / \mathrm{yr}) ; m \& n$ are parameters; and $t=$ stand age $(\mathrm{yr})$.

Secondly, previous empirical studies have suggested that there is an inverse correlation between stand density and stand growth under relaxed interspecific competition [35, 36]. Based on this empirical evidence, we assumed that if the stand density level derived from average $\mathrm{DBH}$, and the stand density plotted on a log-log scale, differed from the full stand density curve, then the growth rate of DBH could be adjusted by this difference.

Based on this assumption, therefore, the lower the stand density relative to the full stand density curve, the larger the upward adjustment of growth rate. By combining formulas (1) and (4), the diameter growth of trees in unthinned stands was expressed using formula (5). In formula (5), the first and second terms show the diameter growth rate according to the stand age, and the growth rate adjustment factor according to the difference between the stand density level and full stand density curve, respectively. Parameter $p$ in formula (5) indicates the degree to which stand density affects the growth rate with respect to DBH. Although previous studies focused on thinned stands managed according to the standard stand density control curve [23], obtained by applying formula (1) to planted forests with classic thinning practices, formula (5) has been applied to thinned stands throughout Japan $[22,37]$. In Japan, thinning from below was common practice in planted forests [24].

$r=m \exp (-n t)+p(K-\log N-d \log D)$

Based on these hypotheses, Fig. (1a) illustrates the overall assumptions embodied in formula (5), showing the relationships between the full density curve, average diameters and stand density and plotted using a log-log scale. Points 1 to 2 and 2 to 4 in Fig. (1) represent the reduction in stand density as a result of self-thinning and the diameter growth after self-thinning, respectively. For example, the move from point 1 to point 2 on the graph represents a decrease in stand density resulting from self-thinning, although the stand age and average tree diameter remain unchanged (Fig. 1). At point 2 the diameter is smaller than that expected according to the full density curve (Fig. 1a), and thus the second term in formula (5) is greater than 0 . Therefore, greater diameter growth rate at point 2 than at point 1 is to be expected because of the reduced competition at point 2 (Fig. 1b). In addition, if there is no self-thinning, the higher growth rate (Fig. 1b) results in trees with diameters larger than would be expected according to the full density curve, as for example at points 3 and 4 in Fig. (1a). In this case, the lower diameter growth rate at point 4 than at point 5 is to be expected (Fig. 1b).

In the present study, parameters $K$ and $d$ were derived from full density curves as mentioned above. By substituting the average DBH growth rate $\left(r_{t}\right)$, stand density $(N)$ and average DBH $(D)$ into formula (5), parameters $m, n$ and $p$ were estimated by minimizing the total squared errors of the observed and calculated growth ratios for the average DBH. The remaining parameters $(m, n, p)$ were estimated by applying the nonlinear least-squares method (the quasiNewton method) to the DBH growth of trees in permanent plots. In order to estimate these parameters from this 
(a)

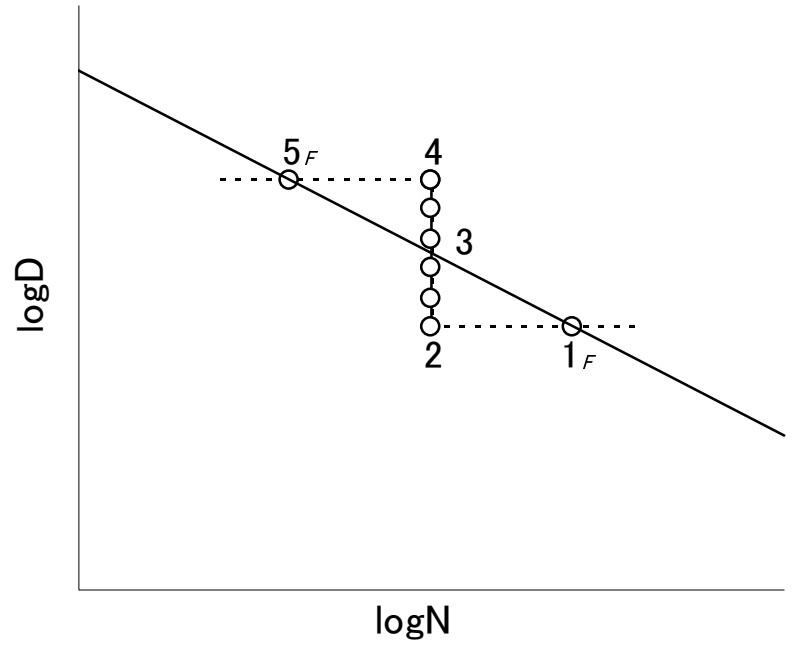

(b)

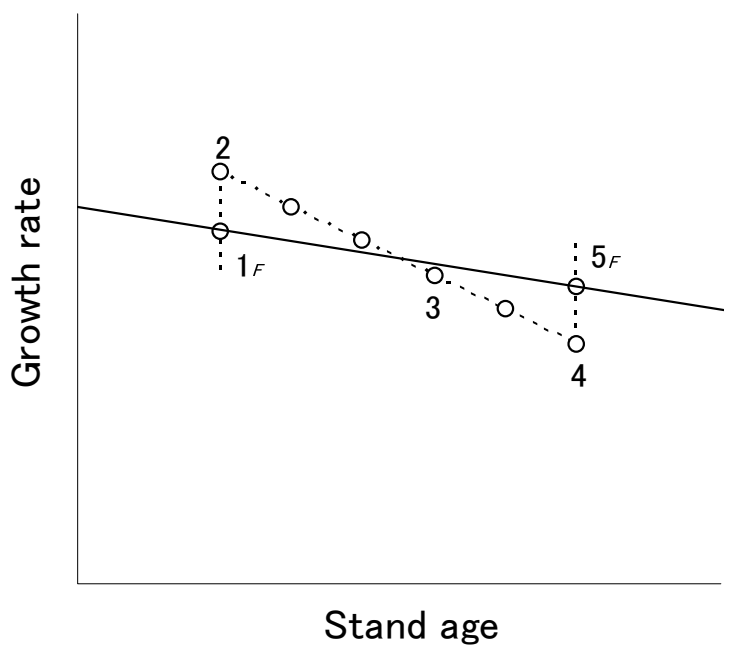

Fig. (1). Examples of (a) stand density depending on self-thinning, and (b) changes in diameter growth rate based on the assumptions underlying the new model. The solid and dotted lines in Fig. (1a) indicate, respectively, the full density curve (formula (1)) and changes in the average diameter $(\mathrm{D}(\mathrm{cm}))$ and stand density $(\mathrm{N}$ (stems/ha)) plotted on a log-log scale. The solid and dotted lines in Fig. (1b) indicate, respectively, the growth rate (formula (4)) under the full density curve and the growth rate depending on the changes in average diameter and stand density shown in Fig. (1a). Each point between 1 and 5 in Fig. (1a, b) represents the same stand age. $F$ indicates the stand density level on the full stand density curve.

procedure, the growth rate of the average DBH (\%/yr) was obtained using formula (6), which gives the geometric mean of the growth rate between two time points in the permanent plots.

$\left.r_{t}=\left(\frac{D_{t+u}}{D_{t}}\right)^{\frac{1}{u}}-1\right) \times 100$

where: $r_{t}=$ growth rate of the average $\mathrm{DBH}$ at age $t(\% / \mathrm{yr}) ; u$ $=$ difference between two measurement time points.

Finally, the accuracy of the estimated stand densities were checked by comparing estimated and observed values in the permanent plots (V1-V4 in Table 1). After confirming the accuracy of the stand density calculated on the basis of the parameters in formula (3), the estimated stand density $(N)$ was substituted into formula (5). The observed $\mathrm{DBH}$, stand density and stand age at the time of the first measurement were used as the initial values for estimating future $\mathrm{DBH}$ and stand density according to formulas (5) and (3). In other words, the observed stand age, density and diameter at the time that the permanent plot was established (A.D.1956-1960) were substituted as initial values into formula (5). The diameter growth was then calculated according to formula (5) to predict the diameter at the time of the next observation. Self-thinning was calculated according to formula (3). These calculations of diameter and self-thinning were repeated until the time of the final observation.

The estimated average diameters were compared with the observed data in the permanent plots. We calculated the error rate by dividing the difference between the observed and estimated values by the observed value. The average error ratio could then be estimated by averaging the absolute value of the all calculated error rates.

We used a Pearson test to check the accuracy of the predicted values derived from formula (5). For this test, $n$ (number of data) was equivalent to the number of measurements per plot (Table 1) and results were considered significant when $p \leq 0.01$. Pearson's correlation coefficients were also calculated in order to check the accuracy of the estimated average DBH derived from the model.

\section{RESULTS AND DISCUSSION}

The relationship between DBH and stand density can be represented by formula (7).

$\log N=4.87-1.19 \log D$

Fig. (2) shows a full density curve derived in a previous study [21], the curve derived from the current work and a standard stand density curve [37] resulting from classic thinning practices in a planted forest region such as the present study area. There are significant differences $(\mathrm{p}<$ 0.05 level after Bonferroni correction) between the standard stand density curve [37], that derived in the current study and the previously derived full density curve [21].

More than half of the stand densities and average DBHs plotted on log-log scale appear above the full density curve derived by Sakaguchi [21]. However, Sakaguchi's [21] data were collected in a number of regions [39-41] and differences between cultivars of Cryptomeria japonica in different localities were not considered, because there were not enough unthinned stand plots at that time. However, in order to analyze the relationships between stand density and average $\mathrm{DBH}$ it would be more rigorous to examine data from a single region. Because the data set used in this study was collected from permanent plots in one region managed as one forest management unit, we consider that formula (7) does express the full stand density and is appropriate for predicting stand growth at the present study site more realistically than the previous full stand density curve.

As shown in Fig. (1), the slope derived from formula (7) was more similar to the standard density curve estimated for 


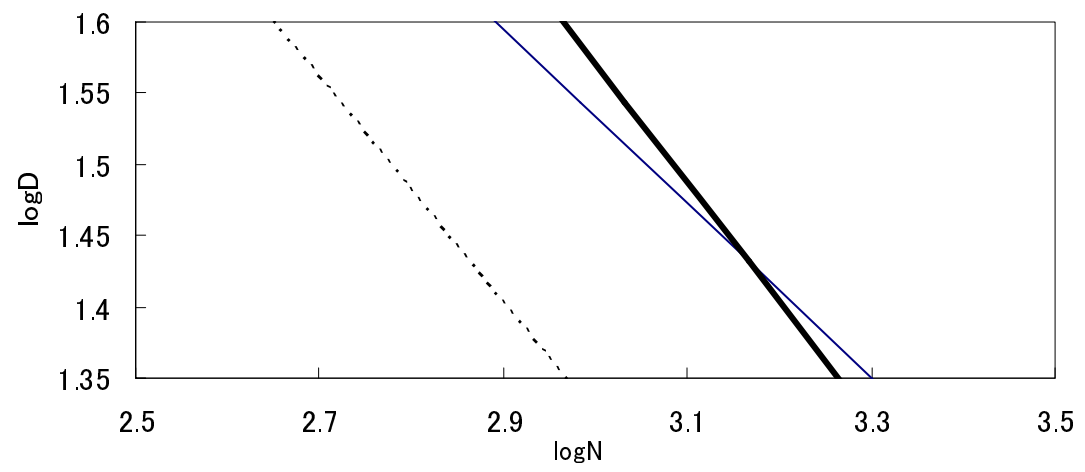

Fig. (2). The full density and stanaara density curves aerived trom previous stuaies and the present one. Bold, fine and dotted lines show, respectively, the full density curve derived in the present study, a full density curve derived from Sakaguchi [21], and a standard density curve derived from Nakajima et al. [27]. $D$ represents DBH $(\mathrm{cm})$, and $N$ represents stand density (stems/ha). The equations for the bold, fine and dotted $\operatorname{lines}$ are $\log N=4.87-1.19 \log D, \log N=5.50-1.63 \log D$, and $\log N=4.67-1.27 \log D$, respectively.

the thinned area [37] than Sakaguchi's full density curve [21]. Previous work has also suggested that the standard density curve has a similar slope to the full density curve $[19,42]$.

Vanderschaaf et al. [14] suggested that, in such situations, a mixed model would be better because it takes into account intracorrelated deviations associated with repeated measurements from permanent plots. However, since density, and probably diameter, did not differ much between the four plots (C1-C4 in Table 1), the results for the parameters of the full density curve $(K$ and $d$ ) will be similar irrespective of the model used. Therefore, it is considered that nonusing a mixed model [14] would be not particularly important in this case.

When estimating the stand density, there appeared to be a large quantity of dead-wood in the high density stands, i.e. those with $R y$ values greater than 0.85 (Fig. 3). An exponential curve fitted to the relationships shown in Fig. (2) resulted in formula (8).

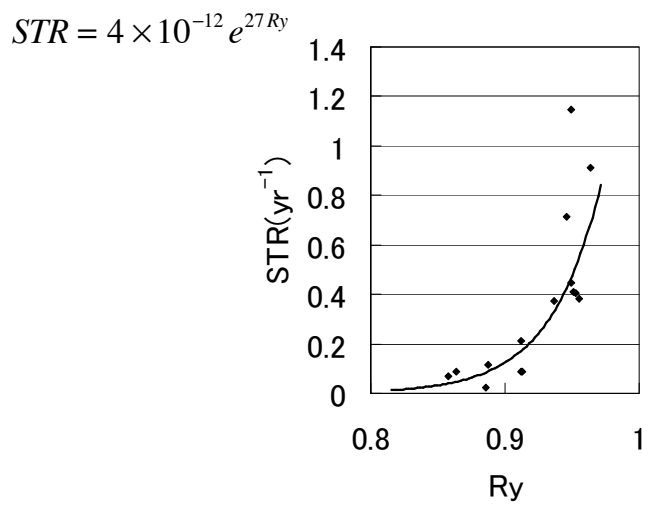

Fig. (3). The relationship between yield index $(R y)$ and selfthinning ratio $(S T R)$. The equation of the exponential curve is $S T R=4 \times 10^{-12} e^{27 R y}$.

The self-thinning model can be expressed as the trajectory of the relationship between $R y$ and the number of trees. This approach, and the self-thinning curve from stand density management diagrams $[16,17]$, both describe deadwood as being dependent on the increase in $R y$ [30]. STR increased as $R y$ increased from 0.85 to 1.0 , with a strong increase above $R y$ values of 0.9 (Fig. 2). In order to minimize the amount of dead-wood in unmanaged forests, it is important to implement thinning in high density stands. The accuracy of the model developed was checked by comparing the estimated and observed values for stand density (Fig. 4). However, because the stand density in plot $\mathrm{V} 1$ almost entirely overlapped that of plot V4, the former is not shown in the figure. The range of error ratios observed in all permanent plots was less than ca. $10 \%$. These results suggest that the decrease in stem numbers observed under the different stand conditions could be modeled by the function of $R y$ derived from existing density management diagrams [31]. As shown in Fig. (4), the higher the initial stand density, the greater the subsequent decrease in stand density. It was confirmed that this tendency of decreasing stand density could be expressed on the basis of the estimated parameters.

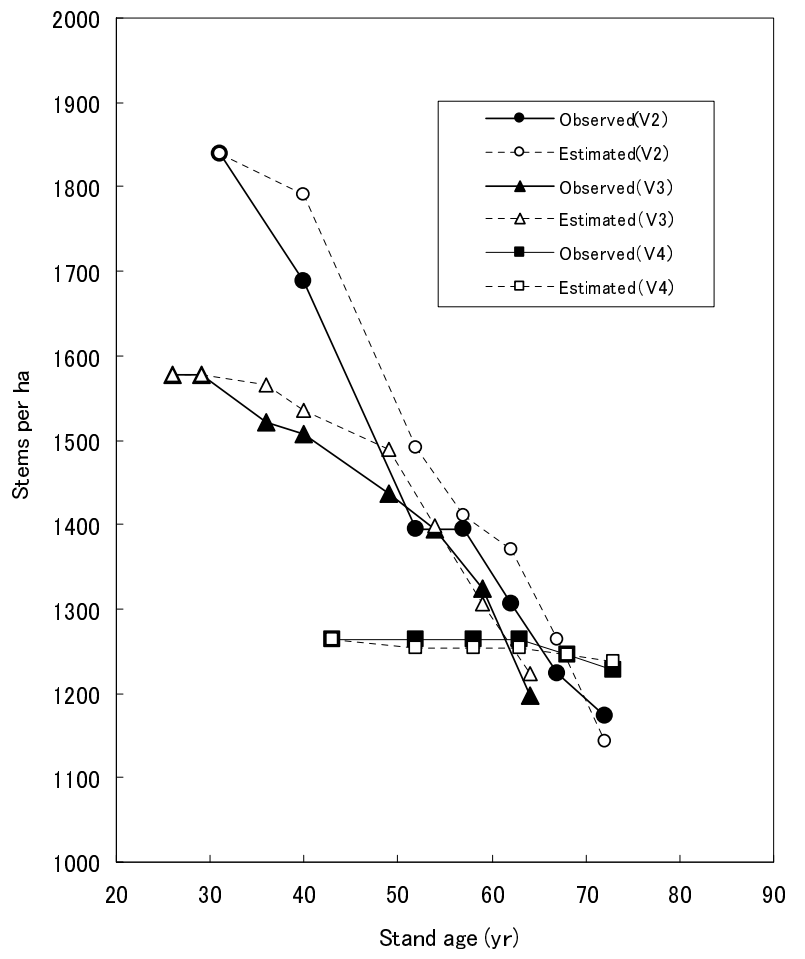

Fig. (4). Comparison between observed and estimated stand density. 
The source of the discrepancy between the observed and the predicted values (Fig. 4) is probably the use of the same parameter values for all the permanent plots. It is possible that the model containing constant parameters would be unable to predict self thinning accurately. For example, a catastrophic natural disturbance in the future could not be predicted by this model. However, because the average error rate of difference between the estimated and observed stand density was between 0.5 and $3.4 \%$ when compared with the permanent plot data, the model could be used to estimate decreasing stand density.

Formula (9), which includes growth parameters for predicting diameter growth, was as follows.

$$
r=1.936 \exp (-0.0169 t)+0.2(4.87-\log N-1.19 \log D)
$$

If the parameter $p$, which reflects the effect of stand density on diameter growth, is positive, this indicates that decreasing stand density has a positive effect on diameter growth. For example, if stand density is below that modeled by the full density curve, the second term in formula (9) will be positive. Therefore, the effect of decreasing stand density on diameter growth will be positive. In this case, the larger the difference between the stand density and the figure based on the full density curve, the greater the effect of stand density on diameter growth. On the other hand, it is possible that stand density is greater than that modeled by the full density curve [21], in which case the second term of formula (9) would be negative. In such a case the effect of stand density on diameter growth would be negative: the larger the difference between the stand density and the figure based on the full density curve, the greater the negative effect of stand density on diameter growth.

Parameter $p$ assumed values between 1.6 and 4.6 for stand density effects on the basis of the standard stand density controls for traditionally thinned stands [22, 37, 43]. In contrast it was only 0.2 when derived from the full density curve, suggesting that decreasing stand density has less effect on the diameter growth rate for the full density curve than for the standard density curve. Generally, stand growth is dependent on tree-crown volume [44, 45]. Furthermore, Zeide [46] has suggested that tree-crown volumes can vary with stand density. Because crown volume under low stand density control, as expressed by standard density curves, is thought to be greater than crown volume under the high stand density control, as expressed by full stand density curves, our finding that parameter $p$ is smaller when diameters are estimated by the full density curve than when estimated by the standard density curve, seems reasonable. In addition, when stand density mainly decreased by controlled the effect could be described by the standard density curve, while density that decreased as a result of selfthinning was best described by the full density curve. With normal, managed thinning, crown growth increases immediately following the formation of canopy gaps. However, with self-thinning, the canopy gap only expands after dead-wood falls or cladoptosis. This difference between the times taken to form significant canopy gaps under normal thinning or self-thinning may be another reason why parameter $p$, and its effect on diameter, was smaller for the full density curve than for the standard density curve.
In order to check the accuracy of the diameter growth predicted using the new model, the estimated diameters were compared with observed values (Fig. 5). The average error rate for the DBH was $2.9 \%$, with a maximum of $8.1 \%$. The squared Pearson's correlation coefficients, indicating the accuracy of the average DBH estimated by the new model, were between 0.97 and 1.00 ( $p$ value $<0.01)$. This confirmed the power of the model for estimating average diameter growth. In the study area, the stand density control diagram and the diameter growth model based on the standard stand density control were developed along with other growth models, produced by the government [31] and other researchers [37]. The absolute error ratios estimated by comparing average diameter calculated on the basis of the stand density control diagram [31] and the growth model based on the standard density curve (Fig. 2) [37] with observed values collected recently from the permanent plots ranged between 17 and $35 \%$ and between 15 and $22 \%$, respectively. By comparing the error rate derived from the new model with that from previous models, we confirmed that the diameter was estimated more successfully by the former (Formula (9)).

These results suggest that increases in average DBH under different stand conditions could be modeled using formula (9). As shown in Fig. (4), the tendency towards increasing average DBH could be expressed using the estimated parameters, suggesting that it might be possible to estimate the future average $\mathrm{DBH}$ growth, using the assumptions mentioned above. Although Guan et al. [1] estimated growth model parameters for each permanent plot, the present study estimated the parameters across all the plots. It may therefore be possible to apply these parameters to diameter growth in other unthinned stands, depending on the initial stand condition.

To predict average DBH growth from formula (9), stand density, average $\mathrm{DBH}$, stand height and stand age are required. In comparison with other process models, formula (9) is easier to use because if requires less data to be collected. For example, stand volume can be calculated by substituting the average DBH and stand height derived from the height growth curve [37], into the stand volume equation [47]. Because the main Japanese carbon sink under the Kyoto protocol [28] is planted forests, including Sugi, it would make sense to estimate the carbon sink using estimated DBH growth by applying formula (9) to other planted forest areas.

Because this study is based on the few unthinned stands that exist, the new model could be applied to high density stands. In addition, because the prediction period in this study was approximately 30 yeas, the new model should be appropriate for predictions over such a length of time. During the prediction period the stands ranged from 25 to 70 years old. The standard rotation period in the target area is around 60 years, so the model needs to be developed further to be useful for predicting the growth of high density stands over this time span.

In this study, we confirmed the hypothesis that average diameter growth could be estimated by using a function incorporating existing $\mathrm{DBH}$, stand density and stand age, with parameters derived from even-aged, monocultures of 

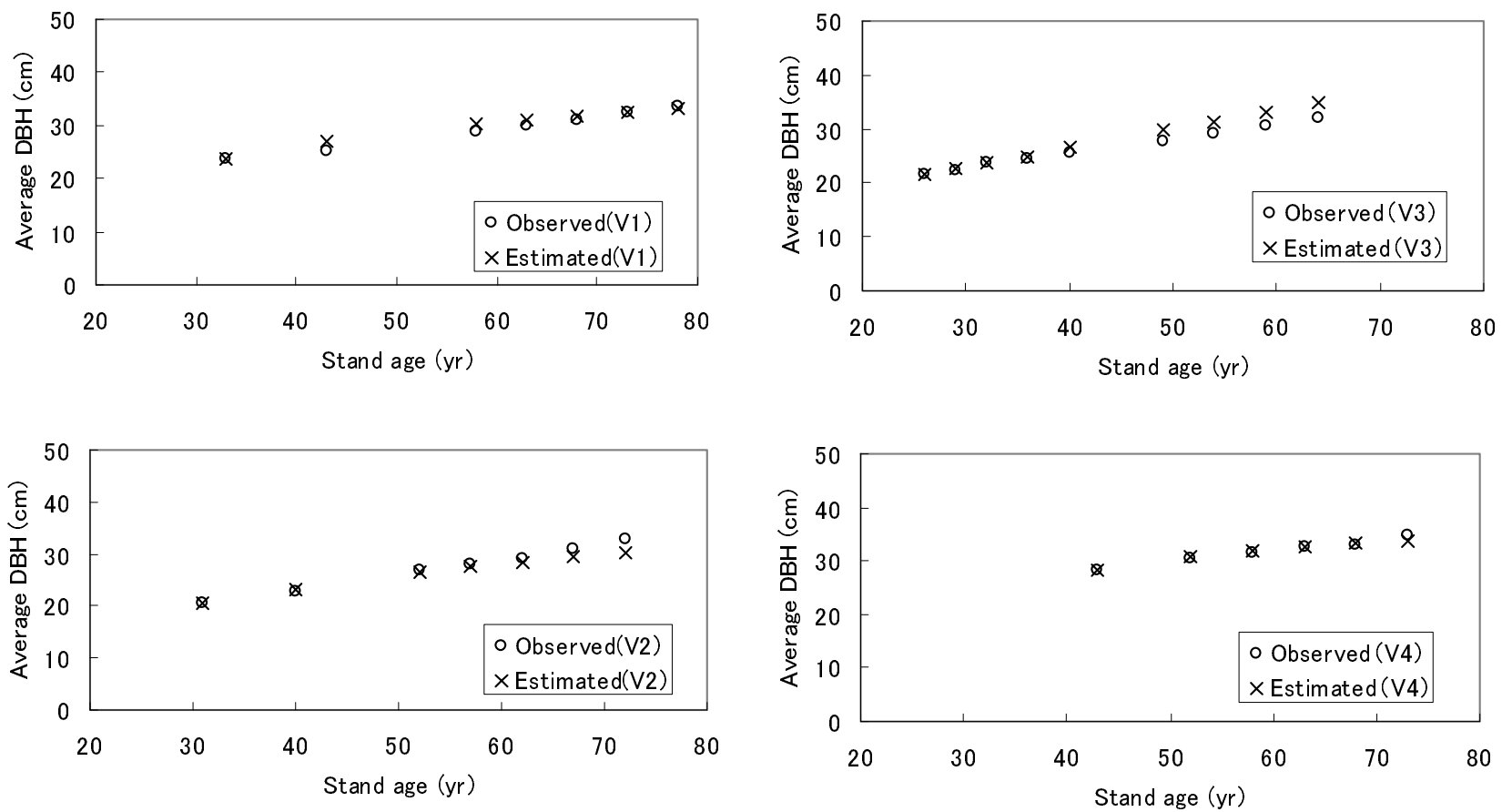

Fig. (5). Comparison between observed and estimated DBH growth. The correlation indexes in each plot are $0.98(\mathrm{p}<0.01), 0.99(\mathrm{p}<0.01)$, $1.00(\mathrm{p}<0.01)$ and $0.97(\mathrm{p}<0.01)$, respectively.

forest trees in high density stands. The estimated parameters for predicting diameter growth in unthinned stands were evaluated by comparing the estimated stand density and $\mathrm{DBH}$, with observed values from the permanent plots. It is possible that this approach to modelling the diameter growth could be applied to other areas by using data collected from other even aged planted tree species and regions in formula (5). Because the main carbon sink in Japan under the Kyoto protocol is planted forests [28], this growth model may be a valuable tool for predicting stand growth. The next challenge is to check the applicability of this growth model to other tree species and regions.

\section{ACKNOWLEDGEMENTS}

We thank the staff of the Tokyo University Forest in Chichibu for their valuable assistance in collecting the data set. This study was supported in part by Research Fellowships from the Japan Society for the Promotion of Science.

\section{CONFLICT OF INTEREST}

The authors declare that they have no conflict of interest.

\section{REFERENCES}

[1] Guan BT, Lin S, Lin Y, Wu Y. Growth efficiency-survivorship relationship and effects of spacing on relative diameter growth rate of Japanese cedars. Forest Ecol Manage 2008; 255: 1713-23.

[2] Poage NJ, Marshall DD, McClellan MH. Maximum Stand-Density Index of 40 western hemlock-Sitka spruce stands in southeast Alaska. West J Appl For 2007; 22: 99-104.
[3] Monserud RA, Ledermann T, Sterba H. Are self-thinning constraints needed in a tree-specific mortality model? For Sci 2004; 50: 848-58.

[4] Pretzsch H, Biber P. A re-evaluation of Reineke's rule and stand density index. For Sci 2005; 51: 304-20.

[5] Toda M, Yokozawa M, Sumida A, et al. Foliage profiles of individual trees determine competition, self-thinning, biomass and NPP of a Cryptomeria japonica forest stand: A simulation study based on a stand-scale process-based forest model. Ecol Model 2009; 220: 2272-80.

[6] Johnsen K, Samuelsson L, Teskey R, et al. Process models as tools in forestry research and management. Forest Sci 2001; 47: 2-8.

[7] Zeide B. Analysis of the $3 / 2$ power law of self-thinning. Forest Sci 1987; 32: 517-37.

[8] Zeide B. Self-thinning and stand density. For Sci 1991; 37: 517-23.

[9] Chen K, Kang H-M, Bai J, et al. Relationship between the virtual dynamic thinning line and the self-thinning boundary line in simulated plant populations. J Integr Plant Biol 2008; 50:280-290.

[10] Hasenauer H, Burkhart HE, Sterba H. Variation in potential volume yield of loblolly pine plantations. For Sci 1994; 40: 162-76.

[11] Hozumi K. Ecological and mathematical considerations on selfthinning in an even-aged pure stands. II. Growth analysis of selfthinning. Bot Mag Tokyo 1980; 93: 149-66.

[12] Jack SB, Long JN. Linkages between silviculture and ecology: an analysis of density management diagrams. For Ecol Manage 1996; 86: 205-20.

[13] Reineke LH. Perfecting a stand-density index for even aged forests. J Agric Res 1933; 46: 627-38.

[14] VanderSchaaf CL, Burkhart HE. Comparison of methods to estimate Reineke's maximum size-density relationship. Forest Sci 2007; 53: 435-42.

[15] Minowa M. A theoretical approach to forest growth modeling. (II) Further discussion on the self-thinning model. J Jpn For Soc 1983; 65: $135-42$.

[16] Ando T. Growth analysis on the natural stands of Japanese red pine (Pinus densiflora Sieb. et Zucc.). II. Analysis of stand density and growth. Bull Gov Forest Stn (Tokyo) 1962; 147: 45-77.

[17] Ando T. Ecological studies on the stand density control in evenaged pure stand. Bull Govt For Exp Sta (Tokyo) 1968; 210:153. 
[18] Stankova TV, Shibuya M. Stand Density Control Diagrams for Scots pine and Austrian black pine plantations in Bulgaria. New Forest 2007; 34: 123-41.

[19] Tadaki Y. The pre-estimating of stem yield based on the competition-density effect. Bull Gov Forest Exp Stn Tokyo 1963; 154:1-19.

[20] Castedo-Dorado F, Crecente-Campo F, Alvarez-Alvarez P, et al. Development of a stand density management diagram for radiata pine stands including assessment of stand stability. Forestry 2009; 82: $1-16$.

[21] Sakaguchi K. Studies on basic factors in thinning. Bull For For Prod Res Inst 1961; 131:1-95.

[22] Shiraishi N. Study on the growth prediction of even-aged stands. Bull Tokyo Univ For 1986; 75: 199-256

[23] Kira T. Density, competition and production in plants. Osaka Government Forest Office, Osaka Miyama 1957; vol. 89: pp. 1-32.

[24] Ohmura K, Sawada H, Oohata S. Growth records on the artificial forest permanent plots in the Tokyo University Forest in Chichibu. Miscellaneous Information, Tokyo University Forests 2004; vol. 43: pp. 1-192.

[25] Soares P, Tome' M. Height-diameter equation for first rotation eucalypt plantations in Portugal. For Ecol Manage 2002; 166: 99109.

[26] Forestry Agency. Annual Report on Trends of Forest and ForestryFiscal Year 2006 Japan Forestry Association, Tokyo 2007.

[27] Nakajima T, Matsumoto $M$, Tatsuhara S. Development and application of an algorithm to estimate and maximize stumpage price based on timber market and stand conditions: A case study of sugi plantations in Gunma Prefecture. J For Plann 2009; 15: 21-7.

[28] Hiroshima T, Nakajima T. Estimation of sequestered carbon in Article-3.4 private planted forests in the first commitment period in Japan. J For Res 2006; 11: 427-37.

[29] Matsumoto M, Hosoda K, Takeuchi M. et al. Development of an accounting method of forest sinks subject to Article 3.4 forest management under the Kyoto Protocol. Mtg Kanto Br Jpn For Soc 2007; 58: 43-44.

[30] Matsumoto M. Grazing in coniferous forests, 2: Estimation of optimal grazing intensity. J Jpn For Soc 1990; 72: 286-91.

[31] Forestry Agency. Stand density management diagram in north Kanto and Higashiyama area. Forestry Agency, Tokyo 1979.

[32] Matsumoto M, Nakajima T. Improvement of LYCS, a program to construct local yield tables. Mtg Kanto Br Jpn For Soc 2006; 57: $69-70$
[33] Pienaar LV, Turnbull KJ. The Chapman-Richards generalization of Von Bertalanffy's growth model for basal area growth and yield in even-aged stands. For Sci 1973; 19: 2-22.

[34] Sweda T, Koide T. Applicability of growth equations to the growth of trees in stem radius. J Jpn For Soc 1981; 63: 113-24.

[35] Kariuki M. Modelling the impacts of various thinning intensities on tree growth and survival in a mixed species eucalypt forest in central Gippsland, Victoria, Australia. For Eco Manage 2008; 256: 2007-17.

[36] Gyenge JE, Fernandez ME, Schlichter TM. Effect of stand density and pruning on growth of ponderosa pines in NW Patagonia, Argentina. Agrofor Syst 2010; 78: 233-41.

[37] Nakajima T, Matsumoto M, Sasakawa $H$, et al. Estimation of growth parameters within the Local Yield Table Construction System for planted forests throughout Japan. J For Plann 2010; 15: 99-108.

[38] Nakagawa T, Koyanagi Y. Analysis of experimental data by the nonlinear least-square method. University of Tokyo Press, Tokyo 1982; p. 206

[39] Akita Regional Forestry Office. Yield table of Japanese sugi in the Akita area. Akita Regional Forestry Office, Akita 1944.

[40] Forestry Agency. Yield table of Japanese sugi in the Ibaraki area. Forestry Agency, Tokyo 1959.

[41] Forestry Agency. Yield table of Japanese sugi in North Kanto Abukuma area. Forestry Agency, Tokyo 1955.

[42] Shidei T. Development of Akamatsu Planted Forests: Basics and Practice. Chikyusha, Tokyo 1963.

[43] Matsumoto M. Construction of yield tables for sugi (Cryptomeria japonica) in Kumamoto district using LYCS. J For Plann 1997; 3 : 55-62.

[44] Kajihara M. Crown-stem size relations in even-aged stands of Cryptomeria japonica. Bull. Kyoto Prefectural Univ Forests 1978; vol. 22: pp. 54-63.

[45] Arain MA, Restrepo-Coupe N. Net ecosystem production in a temperate pine plantation in southeastern Canada. Agric For Meteorol 2005; 128: 223-41.

[46] Zeide B. Fractal analysis of foliage distribution in loblolly pine crowns. Can J For Res 1998; 28 : 106-14.

[47] Forestry Agency. Volume Table-Eastern Japan. Japan Forestry Investigation Committee, Tokyo 1970.

This is an open access article licensed under the terms of the Creative Commons Attribution Non-Commercial License (http://creativecommons.org/licenses/bync/3.0/) which permits unrestricted, non-commercial use, distribution and reproduction in any medium, provided the work is properly cited. 